\title{
Study of participant-spectator matter, thermalization and other related phenomena for neutron-rich colliding pair
}

\author{
Sakshi Gautam ${ }^{1}$ \\ Department of Physics, Panjab University, Chandigarh-160 014, India.
}

\begin{abstract}
We study the participant-spectator matter, density and temperature reached in heavy-ion reactions of neutron-rich systems having $\mathrm{N} / \mathrm{Z}$ ratio varying from 1.0 to 2.0 at 50 and 250 $\mathrm{MeV} /$ nucleon. Our results show a weak dependence of these quantities on the neutron content of the colliding systems. We also shed light on the role of neutron content on the thermalization achieved in a reaction.
\end{abstract}

\footnotetext{
${ }^{1}$ Email: sakshigautm@gmail.com
} 


\section{Introduction}

Heavy-ion collisions (HIC) provide a unique opportunity to study the properties of hot and dense nuclear matter as the nuclear matter is produced at temperature, densities and neutron-proton ratios away from that of the ground state nuclei. The nuclear equation of state (EOS) as well as the in-medium nucleon-nucleon (nn) cross section plays a great role in determining these properties $[1,2]$. The importance of collective transverse in-plane flow in determining these properties has been known for past three decades. The collective transverse in-plane flow has been found to be influenced by the entrance channel parameters like mass of the colliding system [3, 4], impact parameter [5-7] and incident energy [7-9]. The competition between attractive interactions due to mean field (at low incident energies) and repulsive interactions due to nucleon-nucleon (nn) collisions (at high incident energies) led to the disappearance of flow at a particular incident energy (where attractive and repulsive interactions counterbalance each other), known as energy of vanishing flow (EVF) [10]. Since one of the motivations behind studying $\mathrm{HIC}$ is to extract information about the hot and dense nuclear matter, so few studies also shed light on the properties such as density and temperature reached in HIC and also on the participant-spectator matter $[11,12]$. Puri and collaborators have shown that participant-spectator matter can act as a barometer for the study of EVF. The study also pointed towards the insensitivity of participant-spectator matter towards equation of state, momentum-dependent interactions (MDI) and nn cross sections. These studies were done at the EVF. Moreover, these studies were restricted to the systems lying close to the stability line.

With the existing and upcoming radioactive ion beam (RIB) facilities around the world, the present physics interest have been shifted towards nuclei far from the stability line. Various phenomena like fusion-fission, collective flow, EVF and multifragmentation have been studied for such nuclei which lie far from the stability line. So, in the present paper, we aim to see the properties like, density, temperature, thermalization, and participant-spectator matter for neutron-rich systems lying far from the stability line.

The present study is carried out within the framework of isospin-dependent quantum molecular dynamics (IQMD) model which is described in detail in section 2. Our results along with discussions are presented in section 3 and we summarized the results in 
section4.

\section{The model}

The IQMD model [13] which is the extension of quantum molecular dynamics (QMD) [14] model treats different charge states of nucleons, deltas, and pions explicitly, as inherited from the Vlasov-Uehling-Uhlenbeck (VUU) model. The IQMD model has been used successfully for the analysis of a large number of observables from low to relativistic energies. Puri and coworkers have demonstrated that QMD and IQMD carries essential physics needed to demonstrate the various phenomena such as collective flow, multifragmentation and particle production $[15,16]$. The isospin degree of freedom enters into the calculations via symmetry potential, cross sections and Coulomb interactions.

In this model, baryons are represented by Gaussian-shaped density distributions

$$
f_{i}(\vec{r}, \vec{p}, t)=\frac{1}{\pi^{2} \hbar^{2}} \exp \left(-\left[\vec{r}-\overrightarrow{r_{i}}(t)\right]^{2} \frac{1}{2 L}\right) \times \exp \left(-\left[\vec{p}-\overrightarrow{p_{i}}(t)\right]^{2} \frac{2 L}{\hbar^{2}}\right)
$$

where $\mathrm{L}$ is the Gaussian width which can be regarded as a description of the interaction range of a particle. In IQMD the Gaussian width is system dependent. The system dependence of L in IQMD has been introduced in order to obtain the maximum stability of the nucleonic density distribution. for $\mathrm{Au}+\mathrm{Au}$ a value of $\mathrm{L}=8.66 \mathrm{fm}_{2}$ is chosen, for $\mathrm{Ca}+\mathrm{Ca}$ and lighter nuclei $\mathrm{L}=4.33 \mathrm{fm}_{2}$. Nucleons are initialized in a sphere with radius $\mathrm{R}=1.12 \mathrm{~A}^{1 / 3} \mathrm{fm}$, in accordance with liquid-drop model. Each nucleon occupies a volume of $h^{3}$, so that phase space is uniformly filled. The initial momenta are randomly chosen between 0 and Fermi momentum $\left(\vec{p}_{F}\right)$. The nucleons of the target and projectile interact by two- and three-body Skyrme forces, Yukawa potential and Coulomb interactions and momentum-dependent interactions. In addition to the use of explicit charge states of all baryons and mesons, a symmetry potential between protons and neutrons corresponding to the Bethe-Weizsäcker mass formula has been included. The hadrons propagate using Hamilton equations of motion:

$$
\frac{d \overrightarrow{r_{i}}}{d t}=\frac{d\langle H\rangle}{d \overrightarrow{p_{i}}} ; \quad \frac{d \overrightarrow{p_{i}}}{d t}=-\frac{d\langle H\rangle}{d \overrightarrow{r_{i}}}
$$

with 


$$
\begin{aligned}
\langle H\rangle= & \langle T\rangle+\langle V\rangle \\
= & \sum_{i} \frac{p_{i}^{2}}{2 m_{i}}+\sum_{i} \sum_{j>i} \int f_{i}(\vec{r}, \vec{p}, t) V^{\mathrm{ij}}\left(\vec{r}^{\prime}, \vec{r}\right) \\
& \times f_{j}\left(\vec{r}^{\prime}, \vec{p}^{\prime}, t\right) d \vec{r} d \vec{r}^{\prime} d \vec{p} d \vec{p}^{\prime} .
\end{aligned}
$$

The baryon potential $\mathrm{V}^{\mathrm{ij}}$ in the above relation, reads as

$$
\begin{aligned}
V^{i j}\left(\vec{r}^{\prime}-\vec{r}\right)= & V_{\text {Skyrme }}^{i j}+V_{\text {Yukawa }}^{i j}+V_{\text {Coul }}^{i j}+V_{m d i}^{i j}+V_{\text {sym }}^{i j} \\
= & {\left[t_{1} \delta\left(\vec{r}^{\prime}-\vec{r}\right)+t_{2} \delta\left(\vec{r}^{\prime}-\vec{r}\right) \rho^{\gamma-1}\left(\frac{\vec{r}^{\prime}+\vec{r}}{2}\right)\right] } \\
& +t_{3} \frac{\exp \left(\left|\left(\vec{r}^{\prime}-\vec{r}\right)\right| / \mu\right)}{\left(\left|\left(\vec{r}^{\prime}-\vec{r}\right)\right| / \mu\right)}+\frac{Z_{i} Z_{j} e^{2}}{\left|\left(\vec{r}^{\prime}-\vec{r}\right)\right|} \\
& +t_{4} \ln ^{2}\left[t_{5}\left(\vec{p}^{\prime}-\vec{p}\right)^{2}+1\right] \delta\left(\vec{r}^{\prime}-\vec{r}\right) \\
& +t_{6} \frac{1}{\varrho_{0}} T_{3 i} T_{3 j} \delta\left(\vec{r}_{i}^{\prime}-\overrightarrow{r_{j}}\right) .
\end{aligned}
$$

The Skyrme potential $V_{\text {Skyrme }}^{i j}$ consists of two-body and three-body interactions and can be parameterized as:

$$
U=\alpha\left(\frac{\rho}{\rho_{0}}\right)+\beta\left(\frac{\rho}{\rho_{0}}\right)^{\gamma}
$$

The parameters $\alpha$ and $\beta$ can be fixed by requirement that at normal nuclear matter density the average binding energy should be $-16 \mathrm{MeV}$ and total energy should have a minimum at $\rho_{0}$. Different values of $\gamma$ lead to different equation of states. Naturally, larger value of $\gamma$ leads to hard EOS whereas smaller value of $\gamma$ results in soft equation of state. The compressibility $\mathrm{K}(\mathrm{MeV})$ is 200 and 380 for soft and hard EOS, respectively. Here $\mathrm{t}_{6}=100 \mathrm{MeV}$ and $Z_{i}$ and $Z_{j}$ denote the charges of $i$ th and $j$ th baryon, and $T_{3 i}$ and $T_{3 j}$ are their respective $T_{3}$ components (i.e., $1 / 2$ for protons and $-1 / 2$ for neutrons). The parameters $\mu$ and $t_{1}, \ldots, t_{6}$ are adjusted to the real part of the nucleonic optical potential. The Yukawa potential $V_{\text {Yukawa }}^{i j}$ in IQMD is very short ranged $(\mu=0.4 \mathrm{fm})$ and weak. For the density dependence of the nucleon optical potential, standard Skyrme type parametrization is employed. We use a soft equation of state with and without momentum-dependent interactions (MDI) labeled as Soft and SMD, respectively along with the standard isospin- and energy-dependent nn cross section reduced by $20 \%$, i.e. $\sigma=$ 
$0.8 \sigma_{n n}^{\text {free }}$. The cross section for proton-neutron collisions is three times as that for protonproton or neutron-neutron collisions. The cross sections for neutron-neutron collisions are assumed to be equal to the proton-proton cross sections. The more details about the elastic and inelastic cross sections for proton-proton and proton-neutron collisions can be found in $[13,17]$. In a recent study, Gautam et al. [18] has confronted the theoretical calculations of IQMD with the data of ${ }^{58} \mathrm{Ni}+{ }^{58} \mathrm{Ni}$ and ${ }^{58} \mathrm{Fe}+{ }^{58} \mathrm{Fe}$ [19]. The results with the soft EOS (along with the momentum-dependent interactions) and above choice of cross section are in good agreement with the data at all colliding geometries. Two particles collide if their minimum distance $d$ fulfills

$$
d \leq d_{0}=\sqrt{\frac{\sigma_{t o t}}{\pi}}, \sigma_{t o t}=\sigma(\sqrt{s}, \text { type })
$$

where 'type' denotes the ingoing collision partners (N-N...). Explicit Pauli blocking is also included; i.e. Pauli blocking of the neutrons and protons is treated separately. We assume that each nucleon occupies a sphere in coordinate and momentum space. This trick yields the same Pauli blocking ratio as an exact calculation of the overlap of the Gaussians will yield. We calculate the fractions $\mathrm{P}_{1}$ and $\mathrm{P}_{2}$ of final phase space for each of the two scattering partners that are already occupied by other nucleons with the same isospin as that of scattered ones. The collision is blocked with the probability

$$
P_{\text {block }}=1-\left[1-\min \left(P_{1}, 1\right)\right]\left[1-\min \left(P_{2}, 1\right)\right]
$$

and, correspondingly is allowed with the probability $1-\mathrm{P}_{\text {block}}$. For a nucleus in its ground state, we obtain an averaged blocking probability $\left\langle P_{\text {block }}\right\rangle=0.96$. Whenever an attempted collision is blocked, the scattering partners maintain the original momenta prior to scattering.

\section{Results and discussion}

We simulate the reactions of $\mathrm{Ca}+\mathrm{Ca}$ and $\mathrm{Xe}+\mathrm{Xe}$ series having $\mathrm{N} / \mathrm{Z}$ ratio varying from 1.0 to 2.0 in steps of 0.2 . In particular we simulate the reactions of ${ }^{40} \mathrm{Ca}+{ }^{40} \mathrm{Ca},{ }^{44} \mathrm{Ca}+{ }^{44} \mathrm{Ca}$, ${ }^{48} \mathrm{Ca}+{ }^{48} \mathrm{Ca},{ }^{52} \mathrm{Ca}+{ }^{52} \mathrm{Ca},{ }^{56} \mathrm{Ca}+{ }^{56} \mathrm{Ca}$ and

${ }^{60} \mathrm{Ca}+{ }^{60} \mathrm{Ca}$ and ${ }^{110} \mathrm{Xe}+{ }^{110} \mathrm{Xe},{ }^{120} \mathrm{Xe}+{ }^{120} \mathrm{Xe},{ }^{129} \mathrm{Xe}+{ }^{129} \mathrm{Xe},{ }^{140} \mathrm{Xe}+{ }^{140} \mathrm{Xe},{ }^{151} \mathrm{Xe}+{ }^{151} \mathrm{Xe}$, and ${ }^{162} \mathrm{Xe}+{ }^{162} \mathrm{Xe}$ at $\mathrm{b} / \mathrm{b}_{\max }=0.2-0.4$. The incident energies are taken to be 50 and 250 $\mathrm{MeV} /$ nucleon. 


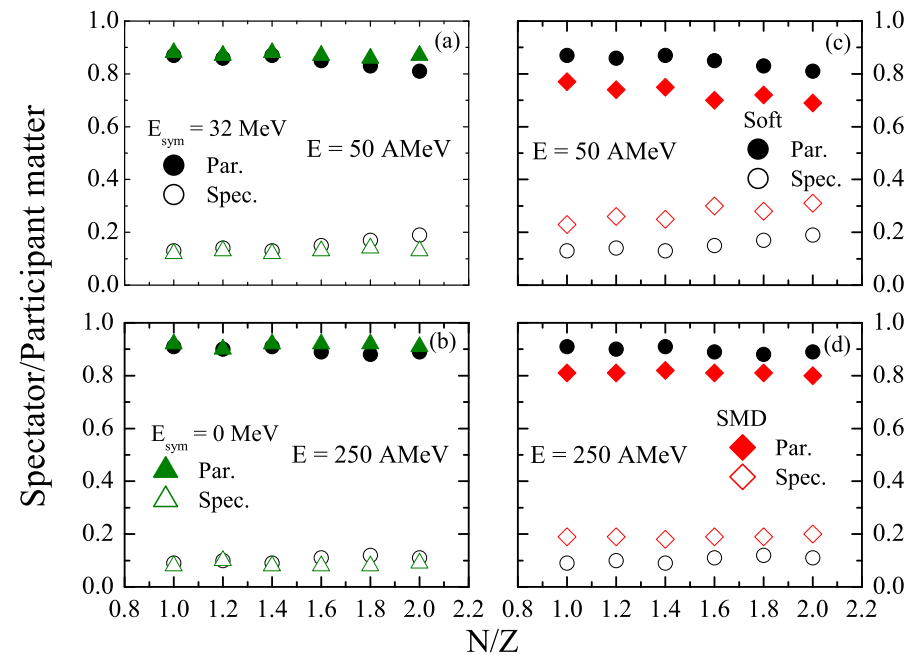

Figure 1: (Color online) The N/Z dependence of participant and spectator matter for the reactions of $\mathrm{Ca}+\mathrm{Ca}$ at 50 (upper panels) and 250 (lower) $\mathrm{MeV} /$ nucleon for different $\mathrm{N} / \mathrm{Z}$ ratios with $\mathrm{E}_{\text {sym }}=0 \mathrm{MeV}$ (left panels) and MDI (right). Various symbols are explained in the text.

In the present work, we define the participant and spectator matter in terms of the nucleonic concept. All the nucleons which have experienced at least one collision are labeled as participant matter, scaled to the total mass of the reacting nuclei. The remaining matter is labeled as spectator matter. These definitions provide a possibility to study the reaction in terms of participant-spectator fireball model.

In fig. 1, we display the $\mathrm{N} / \mathrm{Z}$ dependence of participant-spectator matter for $\mathrm{Ca}+\mathrm{Ca}$ reactions. Upper (lower) panels represent the results for 50 (250) MeV/nucleon. Solid (open) symbols are for participant (spectator) matter. From figure (circles), we find that at lower incident energy there is a slight change in participant-spectator matter with $\mathrm{N} / \mathrm{Z}$ ratio whereas, at higher incident energy of $250 \mathrm{MeV} /$

nucleon, the participant-spectator matter is almost independent of the neutron content of the colliding systems. As we are increasing the neutron content at fixed Z, the mass of the system also increases. Due to increase in mass, the participant matter should increase with $\mathrm{N} / \mathrm{Z}$ ratio (at fixed incident energy). But as we are moving to higher neutron content, the role of symmetry energy also increases, which being repulsive in nature leads to lesser number of collisions in systems with more neutrons and hence participant matter 
should decrease with increase in neutron content of the system. So the net effect is due to the interplay of mass effects and effect of the symmetry energy. To check the relative importance of these two effects we make the strength of potential part of symmetry energy zero and calculate the participant-spectator matter for $\mathrm{Ca}+\mathrm{Ca}$ reactions. The results are displayed in Fig. 1(a) (50 MeV/nucleon) and 1(b) (250 MeV/nucleon) (triangles). From figure, we see that on reducing the strength of symmetry energy to zero, the participant (spectator) matter increases (decreases) slightly for higher neutron content whereas the effect on lower $\mathrm{N} / \mathrm{Z}$ ratios is almost negligible.

The momentum-dependent interactions also play a significant role in the dynamics of heavy-ion collisions. To see the role of MDI on participant-spectator matter we calculate the participant-spectator matter for Soft+MDI (SMD) EOS for the reactions of $\mathrm{Ca}+\mathrm{Ca}$. The results are displayed by diamonds in Fig. 1(c) and 1(d) at 50 and $250 \mathrm{MeV} /$ nucleon, respectively. From figure, we see that participant [closed diamonds] (spectator, open diamonds) matter decreases (increases) with SMD. This is due to the fact that since MDI is repulsive in nature, so the matter is thrown away from the central dense zone and hence lesser number of collisions will take place which leads to decrease (increase) in participant (spectator) matter. Similar behaviour is observed for Xe+Xe reactions (results not shown here).

Since one of the motivations behind studying heavy-ion collisions is to extract information about the hot and dense nuclear matter. In our approach, matter density is calculated by $[20]$

$$
\rho(\vec{r}, t)=\sum_{i=1}^{A_{T}+A_{P}} \frac{1}{(2 \pi L)^{3 / 2}} e^{\left(-\left(\vec{r}-\vec{r}_{i}(t)\right)^{2} / 2 L\right)} .
$$

Here $A_{T}$ and $A_{P}$ stand, respectively, for the mass of the target and projectile. In actual calculations, we take a sphere of $2 \mathrm{fm}$ radius around the center-of-mass and compute the density at each time step during the reaction using Eq. (5.1). Naturally, one can either extract an average density $\left\langle\rho^{a v g}\right\rangle$ over the whole sphere or a maximal value of the density $\left\langle\rho^{\max }\right\rangle$ reached anywhere in the sphere.

In fig. 2, we display the time evolution of maximum (upper panels) and average density (lower panels) at 50 (left panels) and $250 \mathrm{MeV} /$ nucleon (right) for the reactionsof $\mathrm{Ca}+\mathrm{Ca}$. Solid, dashed, dotted, dash-dotted, short dotted and short dash-dotted represent $\mathrm{N} / \mathrm{Z}$ ratios of $1.0,1.2,1.4,1.6,1.8$, and 2.0, respectively. From fig. 2 (left panels), we find that maximum and average density rises as the reaction proceeds, reaches maximum 

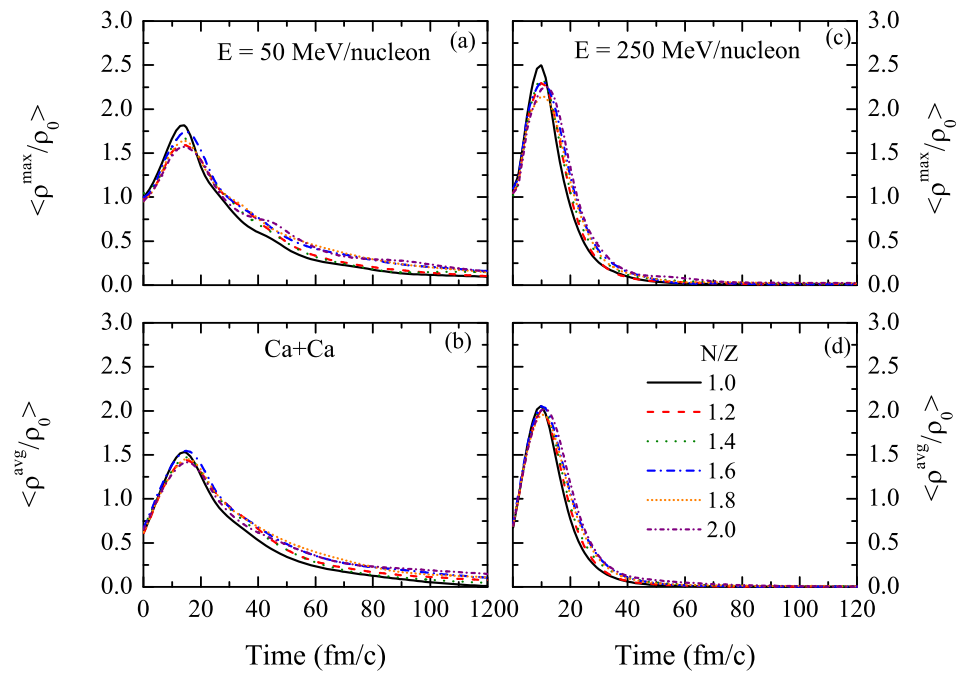

Figure 2: (Color online) The time evolution of the maximum $\left(<\rho_{\max } / \rho_{0}>\right)$ and average $\left(<\rho_{\text {mavg }} / \rho_{0}>\right)$ densities for the reactions of $\mathrm{Ca}+\mathrm{Ca}$ at 50 (left panels) and 250 (right) $\mathrm{MeV} /$ nucleon. Lines have been explained in text.

at $10-20 \mathrm{fm} / \mathrm{c}$ when the matter is highly compressed and finally decreases during the expansion phase. The average density reached in the reactions is about 1.5 times the normal nuclear matter density. The maximal value of the density decreases with increase in neutron content of the system. This slight decrease of the maximal value may be due to the larger effect of symmetry energy in systems with higher neutron content, which in turn, pushes the matter away from the central dense zone due to the repulsive nature of symmetry energy. From fig. 2 (right panels), we see that at $250 \mathrm{MeV} /$ nucleon, much higher density is achieved. This is because of the much violent nature of the reaction at higher incident energy.

In fig. 3, we display the $\mathrm{N} / \mathrm{Z}$ dependence of the maximal value of the maximum and average density achieved in a reaction at 50 (upper panels) and 250 (lower) MeV/nucleon for the reactions of $\mathrm{Ca}+\mathrm{Ca}$ reactions. Solid (open) circles represent the maximal value of $\rho_{\text {avg }}\left(\rho_{\max }\right)$. We find that maximal value almost remains independent of the neutron content of the reacting system.

To see the role of MDI and symmetry energy, we display the results with MDI (Figs. 3(a) and 3(b)) (diamonds) and with no symmetry energy (Figs. 3(c) and 3(d)) (triangles) on average (closed symbols) and maximum density (open symbols) achieved in reactions 


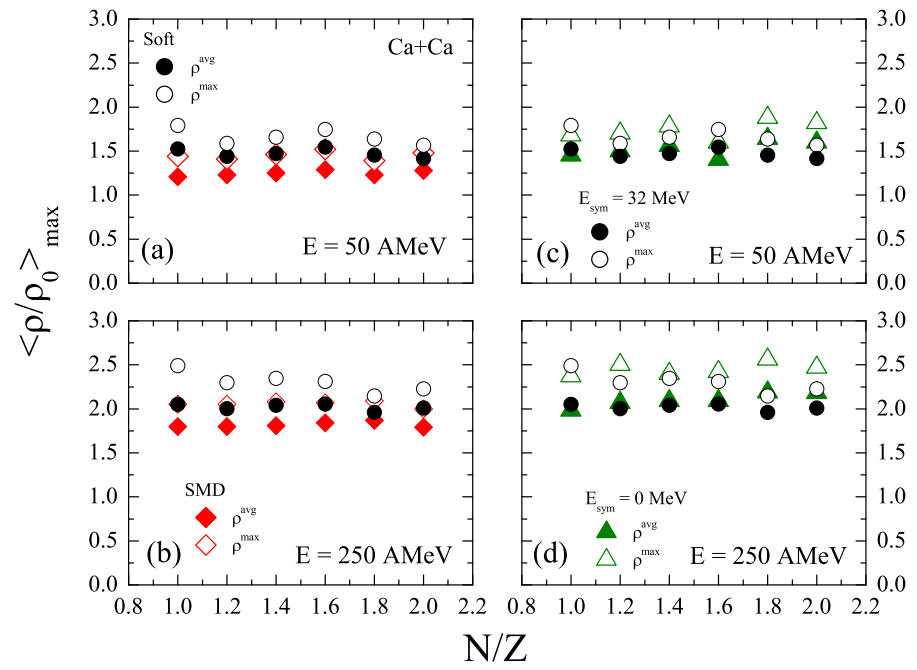

Figure 3: (Color online) The $\mathrm{N} / \mathrm{Z}$ dependence of maximal value of maximum $(<$ $\left.\rho_{\max } / \rho_{0}>\right)$ and average density $\left(<\rho_{\text {avg }} / \rho_{0}>\right)$ for the reactions of $\mathrm{Ca}+\mathrm{Ca}$ at 50 (upper panels) and 250 (lower) $\mathrm{MeV} /$ nucleon with MDI (left panels) and $\mathrm{E}_{\text {sym }}=0 \mathrm{MeV}$ (right). Symbols have same meaning as in Fig. 1.

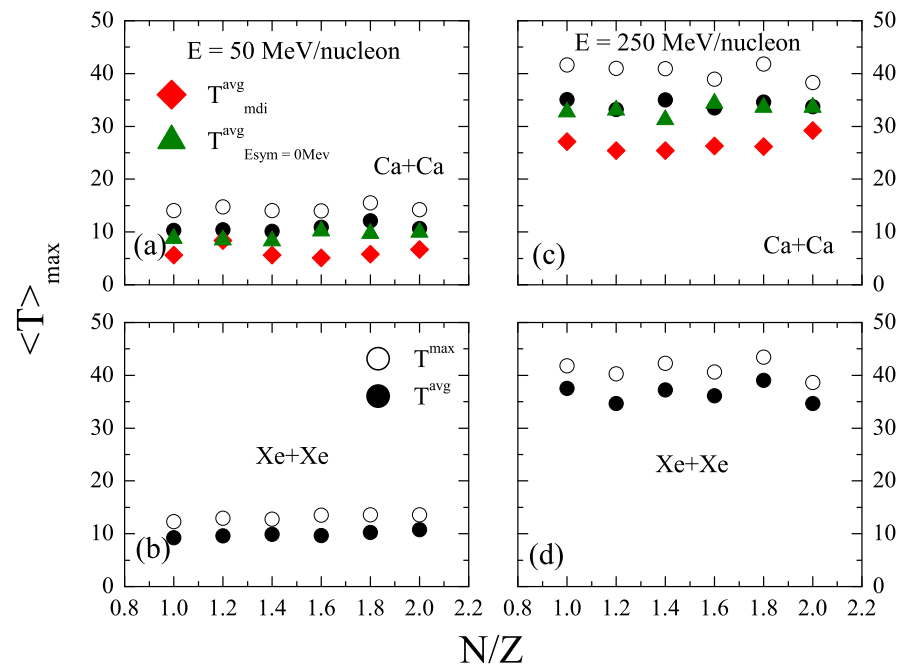

Figure 4: (Color online) The $\mathrm{N} / \mathrm{Z}$ dependence of maximal value of maximum $\left(\mathrm{T}_{\max }\right)$ and average temperature $\left(\mathrm{T}_{\text {avg }}\right)$ for $\mathrm{Ca}+\mathrm{Ca}$ (upper panels) and $\mathrm{Xe}+\mathrm{Xe}$ (lower) at 50 (left) and 250 (right) $\mathrm{MeV} /$ nucleon. Diamonds and triangles represent the results for MDI and $\mathrm{E}_{\text {sym }}=0 \mathrm{MeV}$, respectively. 
of $\mathrm{Ca}+$

Ca. From figure, we see that average and maximum density decreases with SMD as compared to soft EOS, although the N/Z behavior remains independent. Similarly, when we reduce the strength of symmetry energy to zero, the average and maximum density increases slightly for higher neutron content whereas for lower neutron content, the effect of symmetry energy is negligible.

The associated quantity linked with the dense matter is the temperature. In principle, a true temperature can be defined only for a thermalized and equilibrated matter. Since in heavy-ion collisions the matter is non-equilibrated, one can not talk of "temperature". One can, however, look in terms of the local environment only. In our present case, we follow the description of the temperature given in Refs. [20,21]. In the present case, extraction of the temperature $T$ is based on the local density approximation, i.e., one deduces the temperature in a volume element surrounding the position of each particle at a given time step [20,21]. Here, we postulate that each local volume element of nuclear matter in coordinate space and time has some "temperature" defined by the diffused edge of the deformed Fermi distribution consisting of two colliding Fermi spheres, which is typical for a nonequilibrium momentum distribution in heavy-ion collisions.

In this formalism (dubbed the hot Thomas-Fermi approach [20]), one determines extensive quantities like the density and kinetic energy as well as entropy with the help of momentum distributions at a given temperature. Using this formalism, we also extracted the average and maximum temperature within a central sphere of $2 \mathrm{fm}$ radius as described in the case of density.

In fig. 4, we display the $\mathrm{N} / \mathrm{Z}$ dependence of the temperature achieved in a reaction. Upper (lower) panels display the results for $\mathrm{Ca}+\mathrm{Ca}(\mathrm{Xe}+\mathrm{Xe})$ reactions. Solid (open) symbols represent the results of $\mathrm{T}^{\text {avg }}\left(\mathrm{T}^{\max }\right)$. Left (right) panels represent the results of 50 (250) $\mathrm{MeV} /$ nucleon. We find that higher temperatures are achieved in reactions taking place at higher incident energy. For low incident energy, temperature remains almost constant for lower neutron content and shows slight increase for higher neutron content. We also study the effect of MDI (diamonds) and symmetry energy (triangles) on average temperature for the reactions of $\mathrm{Ca}+\mathrm{Ca}$ at 50 and $250 \mathrm{MeV} /$ nucleon. From figure, we see that average temperature decreases for all N/Z ratios when MDI is included. Similarly, when we reduce the strength of symmetry energy to zero, there is a very slight decrease in average temperature. The N/Z behavior, although, remains unaltered. Similar behavior of MDI 

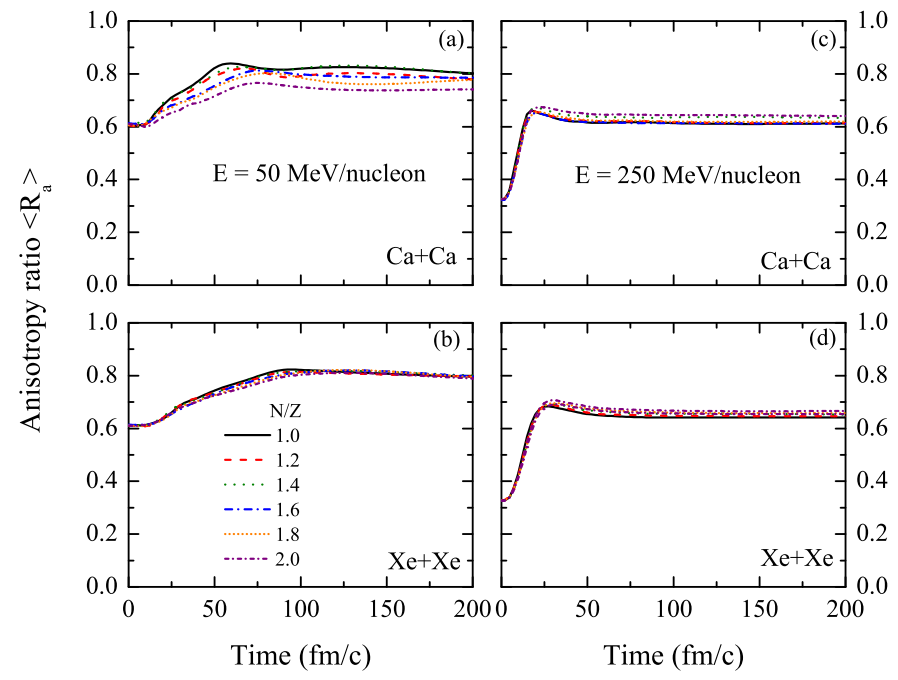

Figure 5: (Color online) The time evolution of anisotropy ratio for $\mathrm{Ca}+\mathrm{Ca}$ (upper panels) and $\mathrm{Xe}+\mathrm{Xe}$ (lower) reactions at 50 (left panels) and 250 (right) $\mathrm{MeV} /$ nucleon for different $\mathrm{N} / \mathrm{Z}$ ratios. Lines have same meaning as in Fig. 2.

and symmetry energy is observed for Xe+Xe reactions (results not shown here).

In fig. 5 we display the time evolution of anisotropy ratio $<R_{a}>$ reached in a reaction. The $<R_{a}>$ is defined as

$$
<R_{a}>=\frac{\sqrt{p_{x}^{2}}+\sqrt{p_{y}^{2}}}{2 \sqrt{p_{z}^{2}}} .
$$

This anisotropy ratio is an indicator of the global equilibrium of the system. This represents the equilibrium of the whole system and does not depend on the local positions. The full global equilibrium averaged over large number of events will correspond to $<R_{a}>$ $=1$. From figure, we see that as the reaction proceeds, the $\left\langle R_{a}\right\rangle$ ratio increases and saturates after the high dense phase is over. We also find that the effect of $N / Z$ ratio is very less on the thermalization achieved in a reaction. We also find a smaller value of $<R_{a}>$ at the start of the reaction for higher energy projectile (see right panels).

In fig. 6, we display the time evolution of relative momentum. The relative momentum $<K_{R}>$ of two colliding Fermi spheres, is defined as

$$
<K_{R}>=<\left|\vec{P}_{P}(\vec{r}, t)-\vec{P}_{T}(\vec{r}, t)\right| / \hbar>
$$



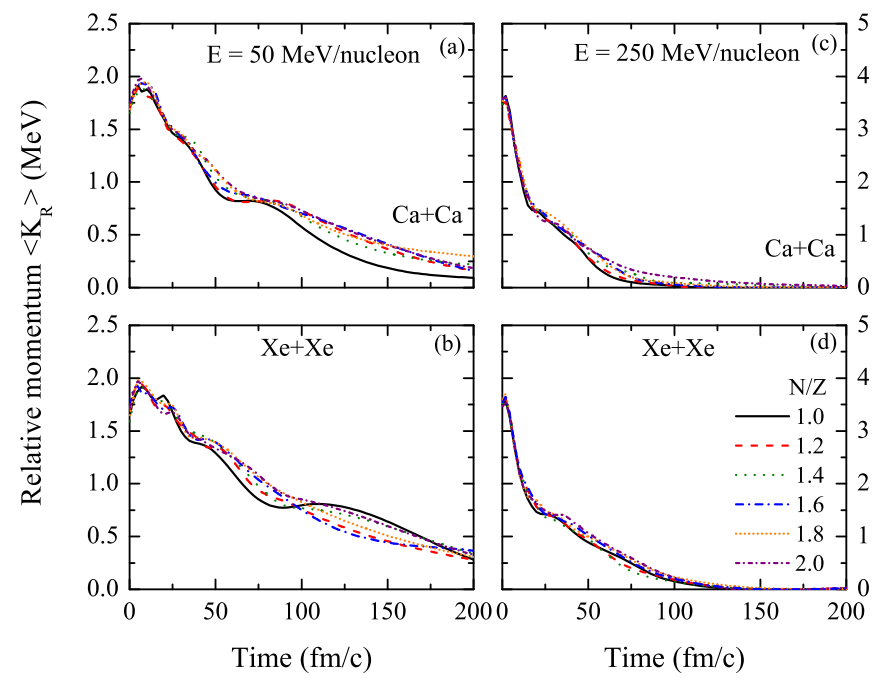

Figure 6: (Color online) Same as Fig. 5, but for the time evolution of relative momentum.

where

$$
\vec{P}_{i}(\vec{r}, t)=\frac{\sum_{j=1}^{A} \vec{P}_{j}(t) \rho_{j}(\vec{r}, t)}{\rho_{j}(\vec{r}, t)} \quad i=1,2,
$$

i.e., $i=1$ and 2 correspond to $i=\mathrm{P}$ (Projectile) and T(target). Here $\vec{P}_{j}$ and $\rho_{j}$ are the momentum and density of the $j$ th particle and $i$ stands for either projectile or target. The $\left\langle K_{R}>\right.$ is an indicator of the local equilibrium because it depends also on the local position $r$.

We find that relative momentum decreases as the reaction proceeds. The effect of neutron content is very less on the value of relative momentum achieved in a reaction. The effect diminishes further at higher incident energy. Also, at higher incident energy, at the start of the reaction we have a higher value of the relative momentum. Also the decrease in $\left\langle K_{R}>\right.$ is much faster for higher incident energy.

\section{Summary}

We studied the dependence of participant-spectator matter, temperature and density reached in heavy-ion reactions of neutron-rich systems on the neutron content of the colliding system. The study is done at 50 and $250 \mathrm{MeV} /$ nculeon. Our calculations showed 
that the behaviour of these quantities is almost independent of the neutron content of the system under study. In addition, we also studied the thermalization achieved in neutron-

rich systems. Thermalization also showed insensitivity towards the neutron content of the reacting systems.

This work has been supported by a grant from Centre of Scientific and Industrial Research (CSIR), Govt. of India. Author is thankful to Profs. J. Aichelin and R. K. Puri for enlightening discussions on the present work.

\section{References}

[1] W. Scheid, H. Müller and W. Greiner, Phys. Rev. Lett. 32, 741 (1974); H. A. Gustafsson et al., Phys. Rev. Lett. 52, 1590 (1984).

[2] K. G. R. Doss et al., Phys. Rev. Lett. 57, 302 (1986).

[3] C. A. Ogilvie et al., Phys. Rev. C 40, 2592 (1989); B. Blättel et al., Phys. Rev. C 43, 2728 (1991).

[4] A. Andronic et al., Phys. Rev. C 67, 034907 (2003).

[5] Q. Pan and P. Danielewicz, Phys. Rev. Lett. 70, 2062 (1993); V. Ramillien et al., Nucl. Phys. A 587, 802 (1995); G. F. Bertsch, W. G. Lynch and M. B. Tsang, Phys. Lett. B 189, 384 (1987).

[6] J. Lukasik et al., Phys. Lett. B 608, 223 (2005).

[7] Y. Zhang and Z. Li, Phys. Rev. C 74, 014602 (2006).

[8] W. M. Zhang et al., Phys. Rev. C 42, R491 (1990); D. Beavis et al., Phys. Rev. C 45, 299( 1992).

[9] J. Lukasik and W. Trautmann, arxiv-0708.2821V1 (2008); B. Hong et al., Phys. Rev. C 66, 034901 (2000).

[10] D. Krofcheck et al., Phys. Rev. Lett. 63, 2028 (1989).

[11] A. D. Sood and R. K. Puri, Phys. Rev. C 70, 034611 (2004). 
[12] A. D. Sood and R. K. Puri, Phys. Rev. C 79, 064618 (2009); ibid. Int. J. Mod. Phys. E 15, 899 (2006).

[13] C. Hartnack et al., Eur. Phys. J. A 1, 151 (1998); C. Hartnack and J. Aichelin, Phys. Rev. C 49, 2801 (1994); S. Kumar et al., ibid. 81, 014611 (2010); ibid. 81, 014601 (2010); V. Kaur et al., Phys. Lett. B597, 612 (2011).

[14] J. Aichelin, Phys. Rep. 202, 233 (1991); Y. K. Vermani et al., J. Phys. G: Nucl. Part. Phys. 36, 105103 (2009); ibid. 37, 015105 (2010); ibid. Eur Phys Lett 85, 62001 (2010); ibid. Phys. Rev. C 79, 064613 (2009), Nucl. Phys A 847, 243 (2010).

[15] J. Dhawan et al., Phys. Rev. C 74, 057901 (2006); ibid. C 74, 054610 (2006); S. Kumar et al., Phys. Rev. C 58, 320 (1998); ibid. C 57, 2744 (1998); ibid. C 78, 064602 (2008).

[16] G. Batko et al., J. Phys. G 20, 461 (1994); ibid. J. Phys. G 22, 131 (1996); E. Lehmann et al., Prog. Part. Nucl. Phys. 30, 219 (1993); S. W. Huang et al., ibid. 30, 105 (1993).

[17] J. Cugnon, T. Mizutani, and J. Vandermeulen, Nucl. Phys. A352, 505 (1981).

[18] S. Gautam, R. Chugh, A. D. Sood, R. K. Puri, C. Hartnack, and J. Aichelin, J. Phys. G: Nucl. Part. Phys. 37, 085102 (2010).

[19] R. Pak et al., Phys. Rev. Lett. 78, 1022 (1997); ibid. 78, 1026 (1997).

[20] D. T. Khoa et al., Nucl. Phys. A542, 671 (1992).

[21] D. T. Khoa et al., Nucl. Phys. A548, 102 (1992); R. K. Puri et al., Nucl. Phys. A575, 733 (1994). 